\title{
SNAIL Induces Epithelial-to-Mesenchymal Transition and Cancer Stem Cell-Like Properties in Aldehyde Dehydroghenase-Negative Thyroid Cancer Cells
}

\author{
Kazuaki Yasui,, Mika Shimamura, ${ }^{1}$ Norisato Mitsutake, ${ }^{2}$ and Yuji Nagayama ${ }^{1}$
}

Background: Epithelial-to-mesenchymal transition (EMT) is thought to play a critical role in the invasion and metastasis of cancer and to be associated with cancer stem cell (CSC) properties. It is not clear if there is a link between EMT and CSCs in thyroid cancers. We therefore investigated the CSC properties of thyroid cancers that underwent EMT.

Method: To induce EMT (spindle-like cell morphology, loss and acquisition of expression of an epithelial marker E-cadherin and a mesenchymal marker vimentin respectively) in an epithelial-type thyroid cancer cell line ACT-1, we used transforming growth factor- $\beta$ (TGF- $\beta$ ), BRAF ${ }^{\mathrm{V} 600 \mathrm{E}}$, and/or Snail homolog 1 (SNAI1, also known as SNAIL). CSC properties were analyzed with assays for cell proliferation, chemosensitivity, in vitro and in vivo tumor formation ability, cell surface antigens, and intracellular aldehyde dehydrogenase (ALDH; a known CSC marker) activities.

Results: EMT was induced most efficiently by SNAIL (ACT-SNAIL cells), whereas TGF- $\beta$ and BRAF ${ }^{\mathrm{V} 600 \mathrm{E}}$ were less efficient. ACT-SNAIL cells showed slightly but significantly enhanced tumor formation ability in an in vitro sphere assay (approximately 3-fold) but not an in vivo subcutaneous tumor growth assay, and showed comparable chemosensitivity compared with the parental ACT-1 cells. However, of interest, although the in vitro sphere-formation ability of $\mathrm{ALDH}^{+}$cells was almost unchanged after SNAIL induction, SNAIL overexpression induced much higher (approximately 14 -fold) spheres in ALDH $^{-}$cells. Thus, ALDH was no longer a CSC marker in ACT-SNAIL cells.

Conclusions: All these data indicate that EMT confers CSC properties in $\mathrm{ALDH}^{-}$cells and appears to influence the ability of ALDH to enrich CSCs.

\section{Introduction}

$\mathrm{T}$ HYROID CANCER IS THE MOST COMMON TYPE of endocrine malignancy, the majority of which arises from thyroid follicular epithelial cells. These cancers are classified into differentiated (DTC; papillary and follicular types), poorly differentiated (PDTC), and anaplastic thyroid cancer (ATC) (1). Although many patients with DTC have excellent overall survival rates, some DTC develop extrathyroidal extension and distant metastases. Further, ATC is one of the most aggressive cancers, with a high mortality rate in humans (2).

Invasion and metastasis have recently been reported to be associated with epithelial-to-mesenchymal transition (EMT) in many cancers including thyroid cancers $(3,4)$. This process is characterized by loss of morphologic features of epithelium, loss of intercellular adhesion molecules (e.g., E-cadherin), gain of mesenchymal markers (vimentin), and high motility and invasive capacities $(4,5)$. Many EMT inducers have been identified, such as various cytokines and transcription factors. A representative in the former group is transforming growth factor- $\beta$ (TGF- $\beta$ ). TGF- $\beta$ is a multifunctional cytokine that acts as a tumor suppressor in early tumor development and promotes tumor cell invasion in later stages (6). TGF- $\beta$ exerts its effect on EMT induction alone or by cooperating with signaling pathways such as RAS and WNT $(7,8)$.

The latter transcription factor group includes SNAIL (SNAI1), SLUG (SNAI2), TWIST, ZEB1, and ZEB2 (9). SNAIL has a key role in EMT both during development and in tumor progression. Previous studies have demonstrated that SNAIL accelerates radiation-induced thyroid carcinoma development (10), and its expression is restricted at the invasive tumor front, showing EMT characteristics in thyroids cancers (11).

Departments of ${ }^{1}$ Molecular Medicine and ${ }^{2}$ Radiation Medical Sciences, Atomic Bomb Disease Institute, Nagasaki University Graduate School of Biomedical Sciences, Nagasaki, Japan. 
In addition, it has recently been shown that certain epithelial cancer cells that pass through EMT acquire cancer stem cell (CSC) properties $(12,13)$. CSCs, or tumor initiating cells, are a small fraction of cancer cells with self-renewal and multipotency, and are important for cancer initiation and maintenance (14). Several attempts have been performed to identify thyroid CSCs using side population, CD133, and aldehyde dehydrogenase (ALDH) activities (15-17). The most reliable marker for thyroid CSCs so far identified appears to be ALDH (17). However, it is not known if there is a link between EMT and CSCs in thyroid cancers, with an exception that Liu et al. have shown, by immunohistochemistry of two thyroid cancer tissues with co-existing DTC and ATC lesions, intense expression of a neural stem cell marker nestin, and loss of E-cadherin expression in ATC areas (18), although there is no definitive evidence for nestin being a thyroid CSC marker.

We therefore investigated the CSC properties of thyroid cancers that underwent EMT. Our results show that EMT seems to be associated with CSC properties in $\mathrm{ALDH}^{-}$human thyroid cancer cells.

\section{Materials and Methods}

\section{Cell cultures}

ACT-1 (19), FRO, KTC-2, and KTC-3 cell lines were originally established from human anaplastic thyroid cancer, and TPC1 cell line from human papillary thyroid cancer. All the cell lines except KTC-3 are in the list of authenticated unique thyroid cancer cell lines (20).

ACT-1 cells were cultured in Dulbecco's modified Eagle's medium (DMEM) supplemented with $10 \%$ fetal bovine serum (FBS) and penicillin/streptomycin (P/S). FRO, KTC-2, KTC-3, and TPC1 cells were cultured in RPMI1640 with 10\% FBS and $\mathrm{P} / \mathrm{S}$. For TGF- $\beta$ treatment, $5 \mathrm{ng} / \mathrm{mL}$ recombinant human TGF- $\beta 1$ (PeproTech, Rocky Hill, NJ) was added to the culture medium every two to three days.

\section{Flow cytometry and fluorescence-activated cell sorting}

$2 \times 10^{6}$ cells were plated in $10 \mathrm{~cm}$ plates, and 24 hours later, the cell suspension was prepared in FACS buffer (PBS/2\% FBS/1 mM EDTA). The cells were stained for 30 minutes at room temperature with anti-CD24-FITC, anti-CD44-PE (both from BD, Heidelberg, Germany), anti-CD133/1-PE (Miltenyi Biotech, Bergisch Gladbach, Germany), anti-CD166-PE, anti-CD90-PE, anti-c-Kit-PE, anti-SSEA-1-PE, anti-CD13FITC, and anti-EpCAM-PE (eBiosciences, San Diego, CA). To test for ALDH1 activity, cells were stained with Aldefluor reagent (Aldagen, Durham, NC) according to the manufacturer's instructions. A specific ALDH inhibitor, diethylaminobenzaldehyde (DEAB), was used as negative control. Samples were analyzed and/or sorted on a FACS Vantage SE System flow cytometer and Cell Quest software (BD Biosciences, San Jose, CA).

\section{Immunofluorescence}

Cells were fixed in 100\% methanol and then permeabilized with acetone for E-cadherin staining, or fixed in $10 \%$ formalin followed by permeabilization with $0.5 \%$ Triton- $X$ for vimentin, Smad2/3, and SNAIL staining. The primary antibodies used were mouse Alexa-488 conjugated anti-Ecadherin (1:500; BD Biosciences), mouse anti-vimentin
(1:100; Dako, Tokyo, Japan), rabbit anti-Smad2/3 (1:125; Cell Signaling, Danvers, MA), and rabbit anti-SNAIL (1:200, Cell Signaling).

Secondary antibodies used were goat anti-mouse or antirabbit coupled to Alexa-488 (Invitrogen, Carlsbad, CA, and Cell Signaling). Cell nuclei were visualized with DAPI (Sigma Aldrich, St. Louis, MO). Slides were mounted with Fluoromount (Diagnostic BioSystems, Pleasanton, CA).

\section{Construction and transductions of retrovirus and lentivirus}

To construct the retroviral vector, human SNAIL cDNA in the pCMV-SPORT6 vector (Origene, Rockville, MD) was digested with XhoI and blunt-ended and then digested with SalI, Released SNAIL cDNA was ligated into the XhoI and HpaI-digested pDON-5Neo (Takara, Otsu, Japan). The resultant vector $\mathrm{pDON}-\mathrm{SNAIL}$ was transfected into the 293GPG packaging cell line (21) and virus-containing supernatants were collected every other day for six days.

A lentivirus coding for mutant BRAF ${ }^{\mathrm{V} 600 \mathrm{E}}$ was previously described (22). Virus infection was done in the presence of $8 \mu \mathrm{g} / \mathrm{mL}$ polybrene. Bulks of transduced cells were obtained after antibiotic selections (G418 for retrovirus and blasticidin for lentivirus) were used (ACT-SNAIL and ACT-BRAF ${ }^{\mathrm{V} 600 \mathrm{E}}$ ). The untransduced, parental ACT-1 cells were used as controls.

\section{Cell viability assays}

Cells were plated in 96-well plates, incubated for 24 hours at $37^{\circ} \mathrm{C}$, and treated with $50 \mu \mathrm{g} / \mathrm{mL}$ 5-FU (Kyowa Hakko Kogyo, Tokyo, Japan), $3 \mu \mathrm{g} / \mathrm{mL}$ doxorubicin (Nippon Kayaku, Tokyo, Japan), or $5 \mathrm{ng} / \mathrm{mL}$ paclitaxel (Nippon Kayaku) for indicated time points. Proliferation of cells was measured using a Cell Counting Kit-8 (Dojindo, Kumamoto, Japan) according to the manufacturer's protocol.

\section{Western blot analysis}

Cells were washed twice with ice-cold $1 \times$ PBS and detached with a scraper. The harvested cells were centrifuged at $3300 \mathrm{~g}$ for 1 minute. The pellet was dissolved in lysis buffer (20 mM Tris pH 7.5, $150 \mathrm{mM} \mathrm{NaCl}, 1 \mathrm{mM}$ EDTA, 0.5\% Triton $\mathrm{X}-100$ ), sonicated, and centrifuged at 13,000 rpm for $10 \mathrm{~min}-$ utes at $4^{\circ} \mathrm{C}$. Protein concentrations were determined according to the method of Bradford (Protein Assay Kit; Bio-Rad, Richmond, CA). Equal amounts $(30 \mu \mathrm{g})$ of proteins per sample were applied to a 7.5-15\% SDS-polyacrylamide separating gel and transferred to a PVDF membrane. The membrane was blocked with $5 \%$ skim milk in $1 \times$ TBS for 1 hour and then immunoblotted with a primary antibody at room temperature for 1 hour. After extensive washing with $1 \times$ TBS with $0.05 \%$ Triton $X-100$, the membranes were incubated with a biotinylated secondary antibody at room temperature for 30 minutes and washed extensively with $1 \times$ TBS $/ 0.05 \%$ Triton X-100. Detection of protein-antibody complexes was performed with streptavidin-horseradish peroxidase (Vectastain ABC kit; Vector Laboratories, Burlingame, CA) and 3,3'-diaminobenzidine $/ \mathrm{H}_{2} \mathrm{O}_{2}$.

The primary antibodies used were rabbit anti-SNAIL (C15D3; 1:1000, Cell Signaling), mouse anti-phospho-ERK1/2 Thr202/Thr204 (E10; 1:2000 Cell Signaling), and mouse $\beta$-actin (C4, 1:1000, Santa Cruz Biotechnology, Santa Cruz, CA). 


\section{Cell migration assay}

The Chemotaxicell Invasion Chamber (Kurabo, Osaka, Japan) was used according to the manufacturer's instructions. $2.5 \times 10^{3}$ cells in serum-free medium were seeded on the interior of the inserts, and the culture medium containing $10 \%$ FBS and $\mathrm{P} / \mathrm{S}$ was added to the lower wells as a chemoattractant. After incubation for the indicated time periods, the cells on the interior of the inserts were removed by swabbing, and the exteriors of the inserts were stained with $1 \%$ crystal violet (Wako, Osaka, Japan) in 10\% ethanol. The cells that had penetrated through the filter were counted under bright-field microscopy.

\section{Sphere-formation assay}

The cells were sorted by FACS and seeded at 1000/well in Ultra Low Attachment 96-well microwell plates (Corning) in DMEM/Ham's F12 (1:1) supplemented with 20 ng/mL EGF (Invitrogen), $20 \mathrm{ng} / \mathrm{mL}$ basic FGF (Invitrogen), and 5\% B27 (Life Technologies Japan, Tokyo, Japan). The numbers of spheres, with a diameter of $100 \mu \mathrm{m}$ or more, were counted every week from week 4 to week 8 .

\section{In vivo tumor growth assay}

Between $1 \times 10^{3}$ and $2.5 \times 10^{5}$ cells $/ 100 \mu \mathrm{L}$ PBS were injected subcutaneously into six-week-old nude mice (Charles River Japan, Shizuoka, Japan). Caliper measurements of tumor volume (length $\times$ width) were conducted every week. Tumor weight was calculated according to the formula: tumor weight $(\mathrm{mg})=$ length $(\mathrm{mm}) \times$ width $(\mathrm{mm}) / 2$. Animal care and all experimental procedures were performed in accordance with the Guideline for Animal Experimentation of Nagasaki University with approval of the Institutional Animal Care and Use Committee.

\section{Statistical analysis}

All data are expressed as mean \pm standard deviation or standard error of the mean. Differences between groups were examined for statistical significance using Student's $t$-test. Statistical significance was set at $p<0.05$.

\section{Results}

\section{EMT status of human thyroid cancer cell lines}

The first series of experiments was performed to investigate the EMT status in five human thyroid cancer cell lines (Fig. 1). ACT-1 cells showed a cobblestone-like shape and expressed an epithelial marker E-cadherin at cell-cell junctions but not a mesenchymal marker vimentin. On the other hand, TPC1, KTC-2, and KTC-3 cells showed a spindle-like shape and expressed vimentin but not E-cadherin. FRO cells contain both cobblestone- and spindle-like cells and expressed E-cadherin (both at cell-cell junctions and in the cytoplasm) and vimentin. These findings demonstrate that ACT-1 cells retain epithelial cell characteristics, TPC1, KTC-2, and KTC-3 have undergone EMT, and FRO cells are intermediate.

\section{Induction of EMT by TGF- $\beta, B R A F^{V 600 E}$, and SNAIL}

To induce EMT in ACT-1 cells, three approaches were employed. The first was exposure to TGF- $\beta$. Even after long-term culture with $5 \mathrm{ng} / \mathrm{mL}$ TGF- $\beta$ (> 2 weeks), ACT- 1 cells remained E-cadherin-positive, and only a small fraction of the cells be- came vimentin-positive (Supplementary Fig. S1A; Supplementary Data are available online at www.liebertpub.com/thy).

Expression of mutant BRAF $\left(\mathrm{BRAF}^{\mathrm{V} 600 \mathrm{E}}\right)$ was used as the second approach because a previous study has shown $\mathrm{BRAF}^{\mathrm{V} 600 \mathrm{E}}$-induced EMT in a normal rat thyroid cell line, PCCL3, via TGF- $\beta$ (23). It has also been reported that in several epithelial cell models, including thyroid cells, the RAF/ ERK/MAPK pathway cooperates with TGF- $\beta$ in the induction of EMT (7). As shown in Supplementary Figure S1A, ACT-1 cells transduced with lentivirus expressing $\mathrm{BRAF}^{\mathrm{V} 600 \mathrm{E}}$ (ACT$\mathrm{BRAF}^{\mathrm{V} 600 \mathrm{E}}$ ) remained E-cadherin-positive and vimentinnegative. However, vimentin expression was clearly visible in ACT-BRAF ${ }^{\mathrm{V} 600 \mathrm{E}}$ cells treated with TGF- $\beta$ for two weeks, although E-cadherin expression remained positive at cell-cell junctions. Thus, the effect of TGF- $\beta$ on EMT induction was incomplete in ACT- 1 and ACT-BRAF ${ }^{\mathrm{V} 600 \mathrm{E}}$ cells.

The integrity of TGF- $\beta$ signaling in ACT-BRAF ${ }^{\mathrm{V} 600 \mathrm{E}}$ cells was confirmed by nuclear translocation of Smad2/3 following TGF- $\beta$ stimulation (Supplementary Fig. S1B), and increased pERK expression confirmed enhanced signal transduction by $\mathrm{BRAF}^{\mathrm{V} 600 \mathrm{E}}$ expression in ACT-BRAF ${ }^{\mathrm{V} 600 \mathrm{E}}$ cells (Supplementary Fig. S1C).

The third approach was overexpression of an EMT-inducing transcription factor. Our preliminary studies with four thyroid cancer cell lines with an EMT phenotype (FRO, TPC1, KTC-2, and KTC-3), in which Western blot and RT-PCR demonstrated extremely low expression levels of SNAIL in FRO and of ZEB1 in FRO and KTC-2 cells (data not shown), did not help us choosing the appropriate transcription factor for EMT induction in thyroid cancer cells. However, as mentioned earlier, SNAIL expression was demonstrated at invasive fronts of thyroid cancer tissues with an EMT phenotype in immunohistochemistry $(10,11)$. Thus, SNAIL was chosen for our study. SNAIL induction of EMT and CSC properties has been described in some other cancers, including breast and squamouscell carcinomas $(12,24)$. Transduction of retrovirus coding for SNAIL at a multiplicity of infection (MOI) of 1:1 only slightly induced vimentin expression but did not affect cell morphology or E-cadherin expression at the cell-cell junctions (Fig. 2A). In contrast, transduction at MOIs of $1: 3$ and 1:10, which induced higher SNAIL expression than that of a MOI of 1:1 (Fig. 2B), completely suppressed E-cadherin expression and induced a fibroblast-like cell morphology and vimentin expression (Fig. 2A). It should be noted here that although the degrees of morphological change and vimentin expression induced by TGF- $\beta$ in ACT-BRAF ${ }^{\mathrm{V} 600 \mathrm{E}}$ cells and those in ACT-SNAIL cells (MOI of 1:3) were similar, complete loss of E-cadherin expression was observed only in ACT-SNAIL cells (MOI of 1:3).

Altogether, overexpression of SNAIL obtained by retrovirus transduction at MOIs of 1:3 or more induced an EMT phenotype in ACT-1 cells most efficiently. We therefore used ACTSNAIL cells (MOI of 1:3; hereafter called ACT-SNAIL cells) for further investigations. These cells showed a higher migration ability—an EMT characteristic — than ACT-1 cells (Fig. 2C).

\section{Characterization of CSC phenotypes in ACT-SNAIL cells}

ACT-SNAIL cells showed reduced (but not significantly different) in vitro proliferation (doubling time 24.2 hours in ACT-1 vs. 28.5 hours in ACT-SNAIL cells) in an in vitro proliferation assay (Fig. 3A). Subcutaneous injection of high 

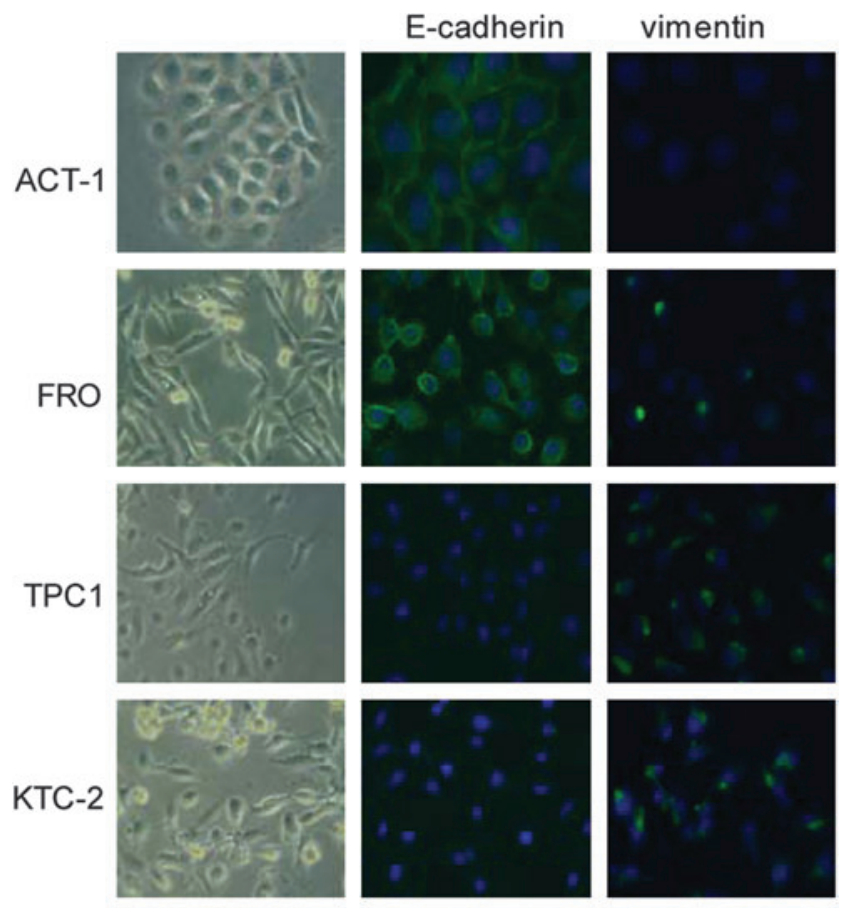

KTC-3
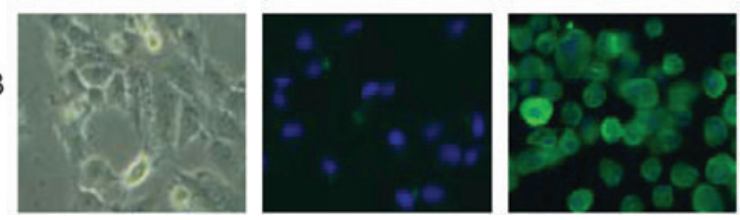

FIG. 1. Morphology and E-cadherin/vimentin expression in five human thyroid cancer cell lines. Phase contrast images (left) and immunofluorescence images for E-cadherin (middle) and vimentin (right) expression in human thyroid cancer cell lines. Five thyroid cancer cell lines were immunostained with antibodies against E-cadherin and vimentin as described in the Materials and Methods section. Magnification: 100×.

numbers of ACT-SNAIL cells $\left(1 \times 10^{5}\right)$ into nude mice readily induced tumors with a growth rate being similar to that in ACT-1 cells (Fig. 3B).

Chemosensitivity of ACT-SNAIL cells was variable, that is, the cells were more resistant to 5 -FU but more sensitive to doxorubicin, as compared to ACT-1 cells (Fig. 3C).

The tumor-initiating ability, estimated by an in vitro sphereformation assay, showed that ACT-SNAIL cells formed approximately 3-fold more spheres relative to ACT-1 cells $(p<0.05)$, although the data were variable (Fig. 3D). However, in in vivo tumor formation assay, the frequency of tumor formation was not significantly different between ACT-1 and ACT-SNAIL cells following subcutaneous injection of different numbers of ACT-1 and ACT-SNAIL cells into nude mice (Table 1). It should be noted here that, although there is an apparent discrepancy between in vitro sphere-formation and in vivo tumor-formation assays, it seems difficult to reproduce such a small (approximately 3-fold) difference detected in an in vitro sphere-formation assay with an in vivo tumor-formation assay.

The cell surface antigens and intracellular ALDH activities, well-known candidate markers for CSCs, were then compared by flow cytometry between ACT-1 and ACT-SNAIL cells. In the cell surface antigen analysis, SNAIL expression
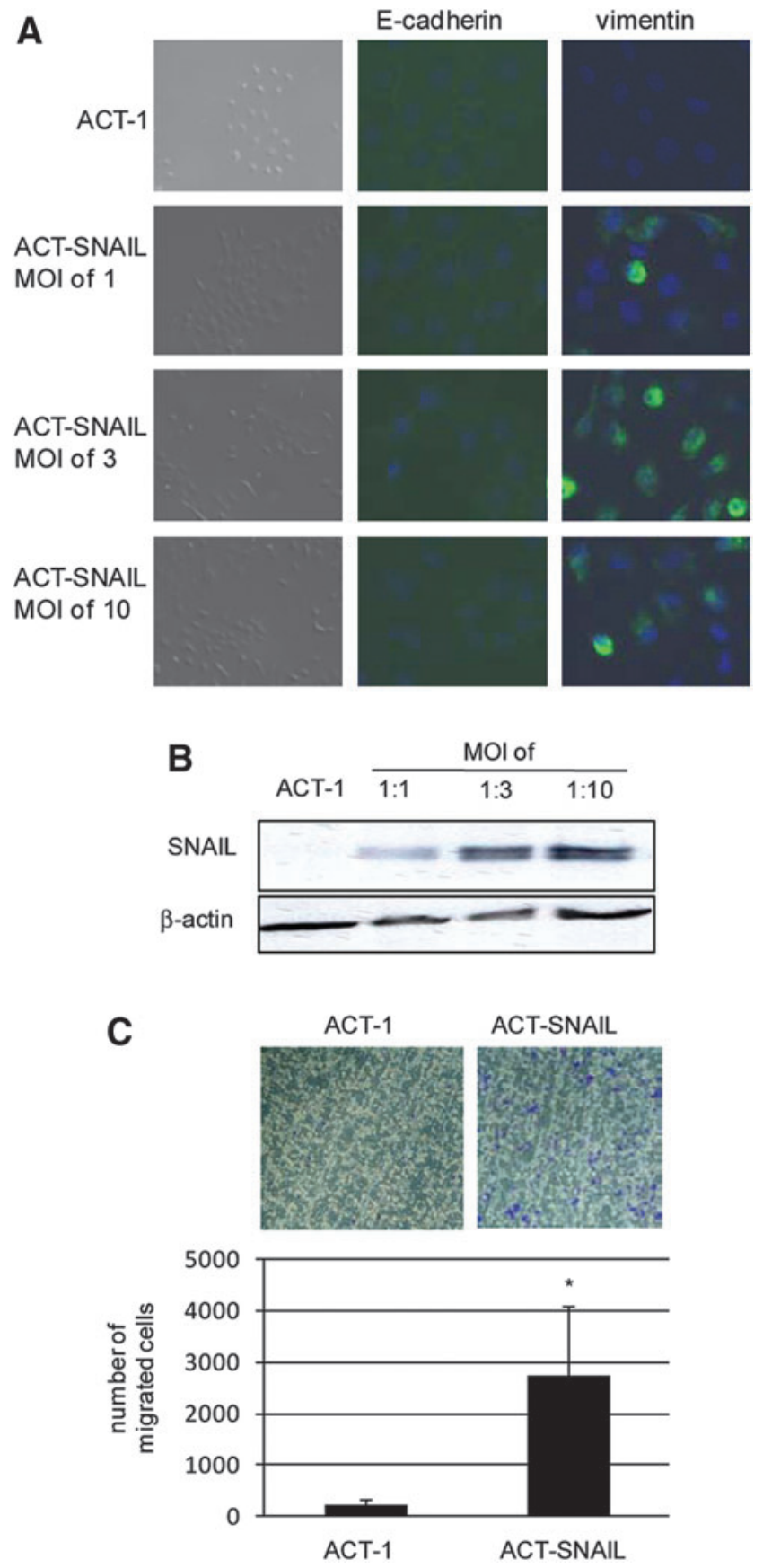

FIG. 2. Induction of epithelial-to-mesenchymal transition (EMT) by SNAIL. (A) Phase contrast images (left) and immunofluorescence images for E-cadherin (middle) and vimentin (right) expression in untransduced, parental ACT-1 and ACTSNAIL cells. (B) Expression of SNAIL in ACT-1 and ACT SNAIL cells. The total cell lysates were subjected to Western blotting using antibody against SNAIL as described in the Materials and Methods section. (C) Cell motility assay in ACT1 and ACT-SNAIL cells with microscopy images of migrated cells (top) and the numbers of migrated cells (bottom). ${ }^{*} p<0.05$. The data are mean \pm standard error of the mean $(n=3)$.

decreased expression of EpCAM and to a lesser extent CD24 (Fig. 4A). However, sphere-forming abilities were very similar between EpCAM ${ }^{\text {high }}$ and EpCAM ${ }^{\text {low }}$ ACT-1, and Ep$\mathrm{CAM}^{\text {high }}$ and EpCAM ${ }^{\text {low }}$ ACT- SNAIL cells (Fig. 4C). In the ALDH assay, 23.4\% parental ACT-1 cells were $\mathrm{ALDH}^{+}$ 

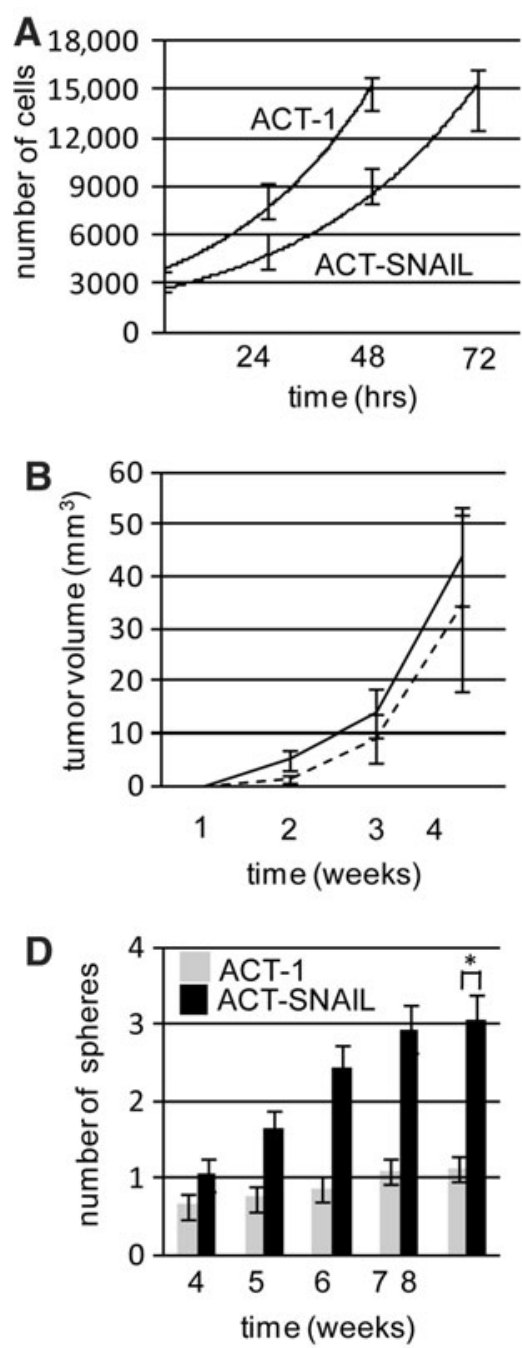

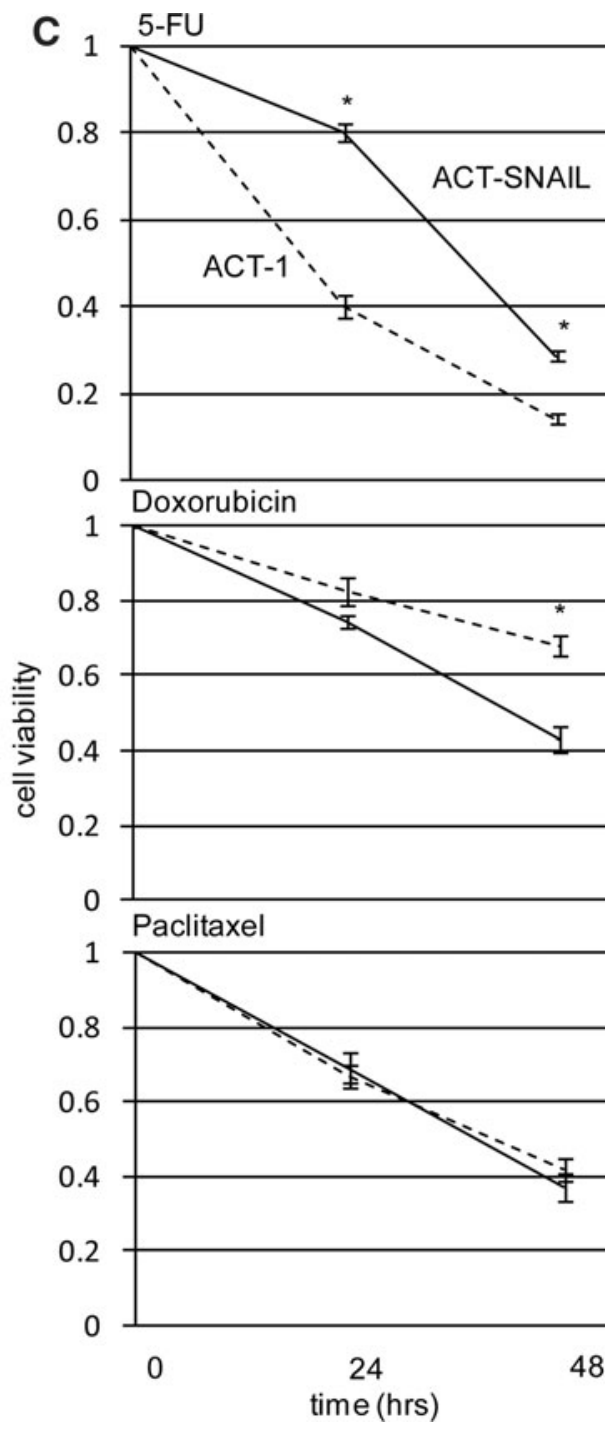

FIG. 3. In vitro and in vivo cell growth, chemosensitivity, and sphere formation in ACT-1 and ACT-SNAIL cells. In vitro cell proliferation (A), in vivo tumor formation (B), chemosensitivity (C), and sphere formation (D) were compared between in ACT-1 and ACT-SNAIL cells as described in the Materials and Methods section. ${ }^{*} p<0.05$. The data are mean \pm standard deviation (A) or standard error of the mean (B-D) $(n=2-6)$.
(Fig. 4B, left), which possessed much higher ability to form spheres than $\mathrm{ALDH}^{-}$ACT-1 cells (Fig. 4D). On the other hand, in ACT-SNAIL cells, the percentage of $\mathrm{ALDH}^{+}$cells decreased (5.2\%), and $\mathrm{ALDH}^{-}$cells conversely showed the significantly higher sphere-forming ability than $\mathrm{ALDH}^{+}$cells. In particular, in vitro sphere-forming ability of $\mathrm{ALDH}^{-}$ACT-SNAIL cells was $\sim 10$ times (6-14 times) as high as that of $\mathrm{ALDH}^{-}$ACT-1 cells, while those of $\mathrm{ALDH}^{+}$ACT-SNAIL and ACT-1 cells were not different. It should be noted here that the EMT status and SNAIL expression levels were not different between $\mathrm{ALDH}^{+}$ and $\mathrm{ALDH}^{-}$cells (Supplementary Fig. S2). Therefore, we would like to interpret these data as meaning that EMT

Table 1. Tumor Incidence of ACT-1 And ACT-SNAIL Cells Injected into Nude Mice

\begin{tabular}{lccccc}
\hline & $1 \times 10^{3}$ & $1 \times 10^{4}$ & $3 \times 10^{4}$ & $1 \times 10^{5}$ & $2.5 \times 10^{5}$ \\
\hline ACT-1 & $0 / 3^{*}$ & $0 / 9$ & $5 / 14$ & $6 / 9$ & $3 / 3$ \\
ACT-SNAIL & $0 / 3$ & $1 / 9$ & $5 / 14$ & $9 / 9$ & $3 / 3$ \\
\hline
\end{tabular}

*Numbers of tumors developed per numbers of tumor cellinjected sites. induced by SNAIL confers CSC properties to $\mathrm{ALDH}^{-}$cells but not to $\mathrm{ALDH}^{+}$cells, which already have CSC properties.

\section{Discussion}

In the present studies, a link between EMT and CSC properties was evaluated in thyroid cancer cell lines. We first found that, among five thyroid cancer cell lines we examined, only ACT-1 cells showed an epithelial phenotype. However, three cell lines-ACT-1, FRO, and KTC-3 cells-were tumorigenic in nude mice and capable of forming nonadherent colonies in an in vitro sphere assay (Fig. 3; Shimamura et al., unpublished data), indicating that all three cell lines with different phenotypes (ACT-1 with an epithelial phenotype, FRO with a mixed phenotype, and KTC-3 with a mesenchymal phenotype) contain CSCs. Next, we demonstrated clear induction of the typical features of EMT, only a slight increase in in vitro sphere-forming (not in vivo tumor-forming) abilities, and no alteration in chemosensitivity by exogenous SNAIL expression in ACT-1 cells. Given that the sphere-forming ability was drastically changed in $\mathrm{ALDH}^{-}$cells but not in $\mathrm{ALDH}^{+}$cells and that the EMT status was not different 
between $\mathrm{ALDH}^{+}$cells and $\mathrm{ALDH}^{-}$cells, a connection between EMT and CSCs seemed to be restricted to $\mathrm{ALDH}^{-}$cells. Although (a) the outcome of suppression of endogenous SNAIL expression cannot be studied in FRO or KTC-3 cells because of their extremely low expression levels of SNAIL and (b) the consequence of SNAIL-induced EMT on CSC properties can only be examined in ACT- 1 cells because this is the only cell line with an epithelial phenotype, all the above data suggest that EMT is linked to CSC properties in $\mathrm{ALDH}^{-}$ ACT-1 thyroid cancer cells.

Expression of E-cadherin, a tumor suppressor (25), has originally been used as a differentiation and prognostic marker of thyroid cancer $(26,27)$, and has later been studied in the context of EMT (28-30). Thus, decreased expression of Ecadherin and/or elevated expression of vimentin in the tissues of ATC compared with DTC and normal tissues have been demonstrated. Therefore, EMT or loss of E-cadherin expression is likely to be related to progression of DTC to ATC (31) rather than a CSC phenotype in the thyroid cancers. Indeed, development of ATC is believed to result largely from the sequential and progressive accumulation of genetic abnormalities - that is, occurrence of p53 mutations mainly in $\mathrm{BRAF}^{\mathrm{V} 600 \mathrm{E}}$ harboring DTC - and p53 is reported to regulate EMT $(32,33)$. The characteristics of ATC are also similar to CSCs: for example, high invasive and metastatic potentials and resistance to chemotherapy. Similar data have also been shown in thyroid-specific $\mathrm{BRAF}^{\mathrm{V} 600 \mathrm{E}}$ transgenic mice, illustrating that EMT is involved in the progression of DTC to PDTC (34).

Little to no effect of TGF- $\beta$ and/or BRAF ${ }^{\mathrm{V} 600 \mathrm{E}}$ on EMT induction contrasts to the previous reports linking these
A

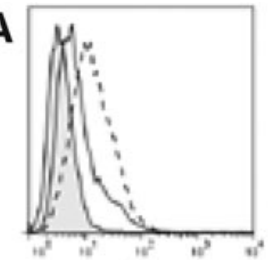

CD224

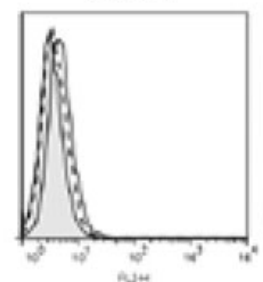

c-Kit

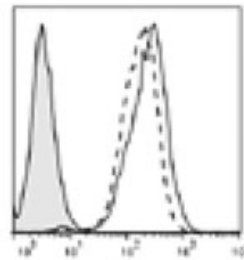

CD44

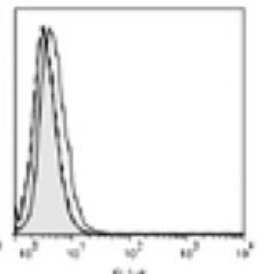

SSEA-1

ACT-1

B
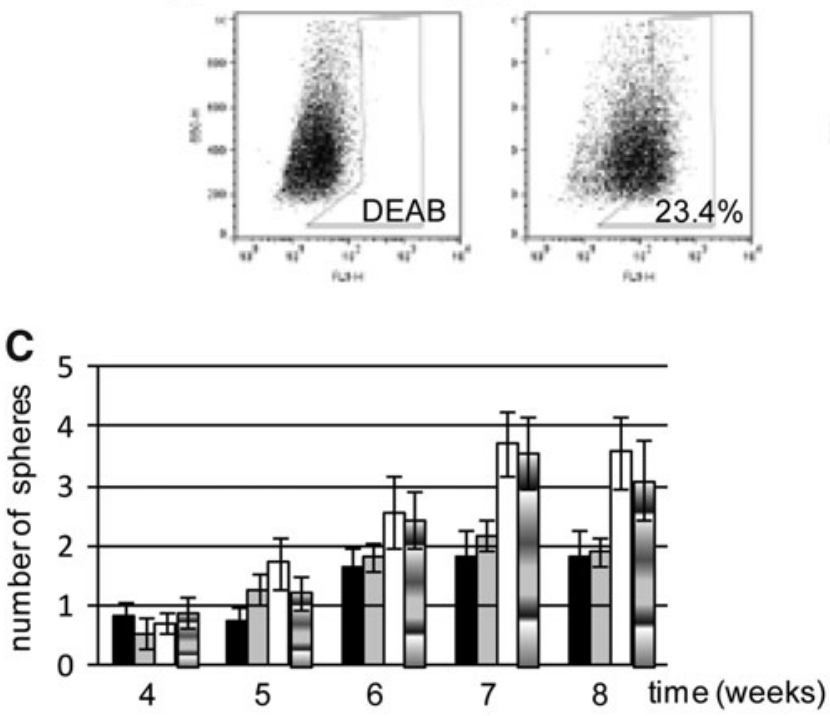

EpCAM ${ }^{\text {high }}$ ACT-1

EpCAM ${ }^{\text {high }}$ ACT-SNAIL

EpCAM ${ }^{\text {low }}$ ACT-1

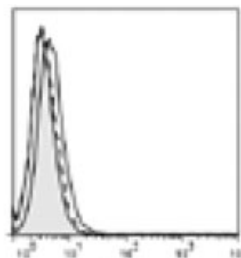

CD133

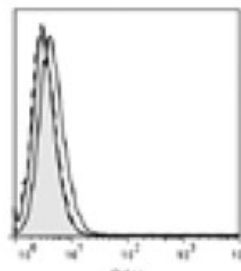

CD13

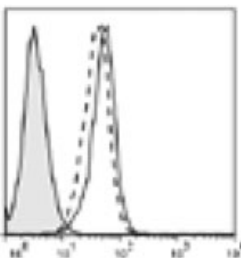

CD166

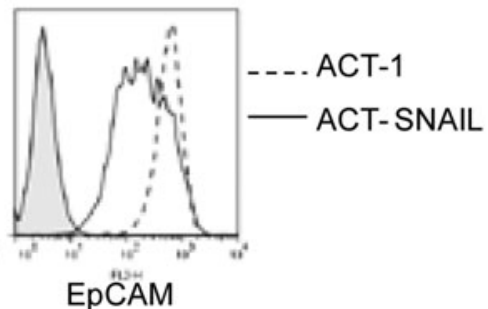

CD90
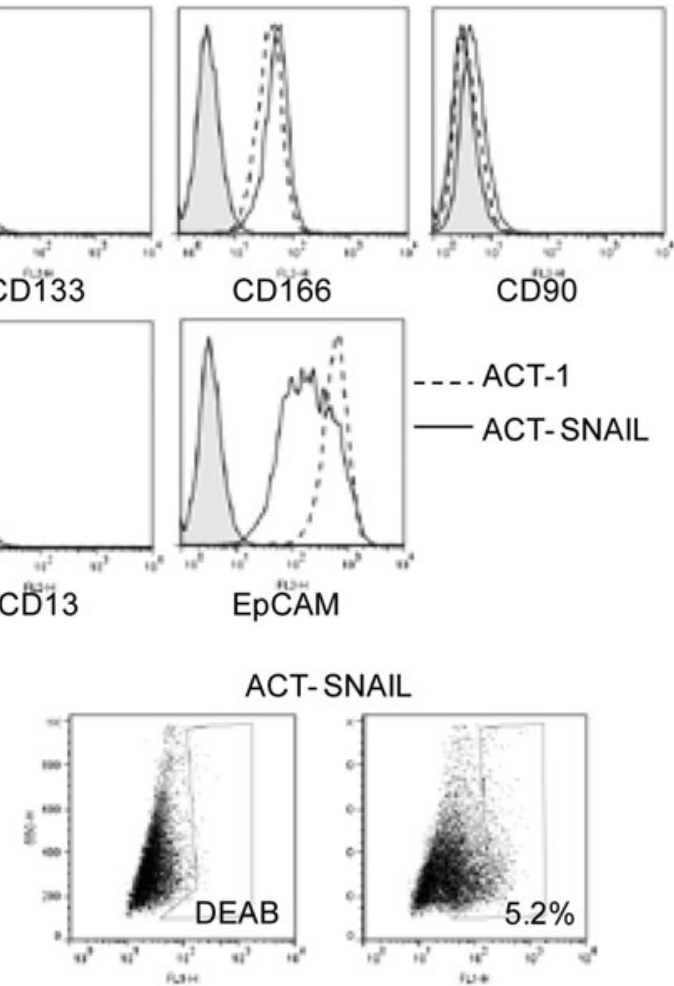

FIG. 4. Comparisons of cell surface markers and aldehyde dehydrogenase (ALDH) activities between ACT-1 and ACTSNAIL cells $(\mathbf{A}, \mathbf{B})$, and of sphere-formation abilities between EpCAM ${ }^{\text {high }}$ versus EpCAM ${ }^{\text {low }}(\mathbf{C}, n=2)$ and $\mathrm{ALDH}^{+}$versus $\mathrm{ALDH}^{-}$cells $(\mathrm{D}, n=4)$. The data are mean \pm standard error of the mean. Statistically significant differences $(p<0.05)$ are indicated: ${ }^{*} \mathrm{ALDH}^{+}$ACT-1 vs. ALDH ${ }^{-}$ACT-1; ${ }^{* *} \mathrm{ALDH}^{-}$ACT-1 vs. ALDH ${ }^{+}$ACT-SNAIL; ${ }^{*} \mathrm{ALDH}^{-} \mathrm{ACT}^{\mathrm{A}} 1$ vs. ALDH ${ }^{-}$ ACT-SNAIL; and ${ }^{\# \#}$ ALDH $^{+}$ACT-SNAIL vs. ALDH ${ }^{-}$ACT-SNAIL. 
molecules with EMT $(23,34)$. Although nuclear translocation of SMAD $2 / 3$ was clearly observed following TGF- $\beta$ stimulation, TGF- $\beta$ transduces the intracellular signal via canonical SMAD-dependent and alternative SMAD-independent pathways. Thus, it is possible that there might be an unknown abnormality in other molecules in the TGF- $\beta$ signaling pathway in ACT-1 cells. Furthermore, since ACT-1 cells have an NRAS mutation (35), interaction of BRAF and mutant NRAS signaling, as previously reported (36), cannot be excluded.

Little expression of SNAIL in thyroid cancer cells with a mesenchymal phenotype (FRO, TPC1, KTC-2, and KTC-3) was somewhat unexpected because of previous reports showing SNAIL expression in thyroid cancer tissues, particularly at their invasive fronts with an EMT phenotype $(10,11)$. However, in addition to SNAIL, other EMT-inducing transcription factors have also been reported to be expressed in thyroid cancers (37), and a recent study has also demonstrated involvement of microRNAs in EMT (28). These molecules may play a role in the EMT status of these cell lines.

Accordingly, expression levels of well-known CSC candidate markers were very similar between the parental ACT-1 and ACT-SNAIL cells, except EpCAM and ALDH. Altered EpCAM expression levels did not affect the efficiency of sphere formation. The decreased expression of EpCAM by SNAIL may be attributed to either suppression of EpCAM gene expression or cleavage (shedding) of EpCAM at the cell surface, as previously reported $(38,39)$. In contrast, the positivity rate and functional property of ALDH was drastically changed by SNAIL expression. Thus, numbers of $\mathrm{ALDH}^{+}$cells decreased while $\mathrm{ALDH}^{-}$cells gained greater sphere-forming ability than $\mathrm{ALDH}^{+}$cells. Given that expression of SNAIL is restricted at the invasive fronts of thyroid cancers with an EMT phenotype (11), the role for ALDH in CSC properties may be dependent on the EMT status, that is, ALDH may be a good CSC candidate marker in the non-EMT area but not in the EMT area, although ALDH has recently been reported to be a CSC marker in thyroid cancers (or able to enrich CSCs) (17). Of interest, Biddle et al. have recently demonstrated unidirectional conversion of $\mathrm{EMT}^{+} \mathrm{ALDH}^{+}$squamous CSCs to $\mathrm{EMT}^{+} \mathrm{ALDH}^{-}$squamous-cell CSCs, although its functional significance remained obscure (40).

In conclusion, in the present studies, we show an association between EMT and CSC properties in $\mathrm{ALDH}^{-}$thyroid cancer cells. Furthermore, our results showing the loss of the ability of ALDH to enrich CSCs in ACT-SNAIL cells suggest that EMT may influence the usefulness of ALDH as a CSC marker. Further studies are necessary to clarify the relationship between EMT, CSCs, and ALDH in thyroid cancer.

\section{Acknowledgement}

We thank Dr. Naoyoshi Onoda (Osaka City University Graduate School of Medicine, Osaka, Japan) for providing ACT-1 cells, which were originally established by Dr. Seiji Ohata (Tokushima University, Tokushima, Japan).

\section{Author Disclosure Statement}

The authors declare that no competing financial interests exist.

\section{References}

1. Kondo T, Ezzat S, Asa SL 2006 Pathogenetic mechanisms in thyroid follicular-cell neoplasia. Nat Rev Cancer 6: 292-306.

2. Sipos J, Mazzaferri E 2010 Thyroid cancer epidemiology and prognostic variables. Clin Oncol 22:395-404.

3. Floor S, van Staveren WC, Larsimont D, Dumont JE, Maenhaut C 2011 Cancer cells in epithelial-to-mesenchymal transition and tumor-propagating-cancer stem cells: distinct, overlapping or same populations. Oncogene 30:4609-4621.

4. Polyak K, Weinberg RA 2009 Transitions between epithelial and mesenchymal states: acquisition of malignant and stem cell traits. Nat Rev Cancer 9:265-273.

5. Thiery JP, Sleeman JP 2006 Complex networks orchestrate epithelial-mesenchymal transitions. Nat Rev Mol Cell Biol 7:131-142.

6. Miyazono K 2009 Transforming growth factor-beta signaling in epithelial-mesenchymal transition and progression of cancer. Proc Jpn Acad Ser B Phys Biol Sci 85:314.

7. Janda E, Lehmann K, Killisch I, Jechlinger M, Herzig M, Downward J, Beug H, Grünert S 2002 Ras and TGF $\beta$ cooperatively regulate epithelial cell plasticity and metastasis. J Cell Biol 156:299-314.

8. Scheel C, Eaton EN, Li SHJ, Chaffer CL, Reinhardt F, Kah KJ, Bell G, Guo W, Rubin J, Richardson AL 2011 Paracrine and autocrine signals induce and maintain mesenchymal and stem cell states in the breast. Cell 145:926-940.

9. Peinado H, Olmeda D, Cano A 2007 Snail, Zeb and bHLH factors in tumour progression: an alliance against the epithelial phenotype? Nat Rev Cancer 7:415-428.

10. Hardy RG, Vicente-Duenas C, Gonzalez-Herrero I, Anderson C, Flores T, Hughes S, Tselepis C, Ross JA, Sanchez-Garcia I 2007 Snail family transcription factors are implicated in thyroid carcinogenesis. Am J Pathol 171:1037-1046.

11. Vasko V, Espinosa AV, Scouten W, He H, Auer H, Liyanarachchi S, Larin A, Savchenko V, Francis GL, de la Chapelle A, Saji M, Ringel MD 2007 Gene expression and functional evidence of epithelial-to-mesenchymal transition in papillary thyroid carcinoma invasion. Proc Natl Acad Sci USA 104:2803-2808.

12. Mani SA, Guo W, Liao MJ, Eaton EN, Ayyanan A, Zhou AY, Brooks M, Reinhard F, Zhang CC, Shipitsin M 2008 The epithelial-mesenchymal transition generates cells with properties of stem cells. Cell 133:704-715.

13. Kong D, Banerjee S, Ahmad A, Li Y, Wang Z, Sethi S, Sarkar FH 2010 Epithelial to mesenchymal transition is mechanistically linked with stem cell signatures in prostate cancer cells. PLoS One 5:e12445.

14. Clarke MF, Dick JE, Dirks PB, Eaves CJ, Jamieson CH, Jones DL, Visvader J, Weissman IL, Wahl GM 2006 Cancer stem cells-perspectives on current status and future directions: AACR workshop on cancer stem cells. Cancer Res 66:93399344.

15. Mitsutake N, Iwao A, Nagai K, Namba H, Ohtsuru A, Saenko V, Yamashita S 2007 Characterization of side population in thyroid cancer cell lines: cancer stem-like cells are enriched partly but not exclusively. Endocrinology 148: 1797-1803.

16. Friedman S, Lu M, Schultz A, Thomas D, Lin RY 2009 CD133 + anaplastic thyroid cancer cells initiate tumors in immunodeficient mice and are regulated by thyrotropin. PLoS One 4:e5395.

17. Todaro M, Iovino F, Eterno V, Cammareri P, Gambara G, Espina V, Gulotta G, Dieli F, Giordano S, De Maria R, Stassi 
G 2010 Tumorigenic and metastatic activity of human thyroid cancer stem cells. Cancer Res 70:8874-8885.

18. Liu J, Brown RE 2010 Immunohistochemical detection of epithelialmesenchymal transition associated with stemness phenotype in anaplastic thyroid carcinoma. Int J Clin Exp Pathol 3:755-762.

19. Chung SH, Onoda N, Ishikawa T, Ogisawa K, Takenaka C, Yano Y, Hato F, Hirakawa K 2002 Peroxisome proliferatoractivated receptor gamma activation induces cell cycle arrest via the p53-independent pathway in human anaplastic thyroid cancer cells. Jpn J Cancer Res 93:1358-1365.

20. Schweppe RE, Klopper JP, Korch C, Pugazhenthi U, Benezra M, Knauf JA, Fagin JA, Marlow LA, Copland JA, Smallridge RC, Haugen BR 2008 Deoxyribonucleic acid profiling analysis of 40 human thyroid cancer cell lines reveals crosscontamination resulting in cell line redundancy and misidentification. J Clin Endocrinol Metab 93:4331-4341.

21. Ory DS, Neugeboren BA, Mulligan RC 1996 A stable humanderived packaging cell line for production of high titer retrovirus/vesicular stomatitis virus G pseudotypes. Proc Natl Acad Sci USA 93:11400-11406.

22. Matsuse M, Mitsutake N, Tanimura S, Ogi T, Nishihara E, Hirokawa M, Fuziwara CS, Saenko VA, Suzuki K, Miyauchi A, Yamashita S 2013 Functional characterization of the novel BRAF complex mutation, BRAF(V600delinsYM), identified in papillary thyroid carcinoma. Int J Cancer 132:738-743.

23. Riesco-Eizaguirre G, Rodriguez I, De la Vieja A, Costamagna E, Carrasco N, Nistal M, Santisteban P 2009 The BRAFV600E oncogene induces transforming growth factor beta secretion leading to sodium iodide symporter repression and increased malignancy in thyroid cancer. Cancer Res 69:8317-8325.

24. Zhu LF, Hu Y, Yang CC, Xu XH, Ning TY, Wang ZL, Ye JH, Liu LK 2012 Snail overexpression induces an epithelial to mesenchymal transition and cancer stem cell-like properties in SCC9 cells. Lab Invest 92:744-752.

25. Wong AS, Gumbiner BM 2003 Adhesion-independent mechanism for suppression of tumor cell invasion by Ecadherin. J Cell Biol 161:1191-1203.

26. Brabant G, Hoang-Vu C, Cetin Y, Dralle H, Scheumann G, Molne J, Hansson G, Jansson S, Ericson LE, Nilsson M 1993 E-cadherin: a differentiation marker in thyroid malignancies. Cancer Res 53:4987-4993.

27. Scheumman GF, Hoang-Vu C, Cetin Y, Gimm O, Behrends J, von Wasielewski R, Georgii A, Birchmeier W, von Zur Muhlen A, Dralle H, et al. 1995 Clinical significance of E-cadherin as a prognostic marker in thyroid carcinomas. J Clin Endocrinol Metab 80:2168-2172.

28. Braun J, Hoang-Vu C, Dralle H, Huttelmaier S 2010 Downregulation of microRNAs directs the EMT and invasive potential of anaplastic thyroid carcinomas. Oncogene 29:4237-4244.

29. Wiseman SM, Masoudi H, Niblock P, Turbin D, Rajput A, Hay J, Filipenko D, Huntsman D, Gilks B 2006 Derangement of the E-cadherin/catenin complex is involved in transformation of differentiated to anaplastic thyroid carcinoma. Am J Surg 191:581-587.

30. Aratake Y, Nomura H, Kotani T, Marutsuka K, Kobayashi K, Kuma K, Miyauchi A, Okayama A, Tamura K 2006 Coexistent anaplastic and differentiated thyroid carcinoma: an immunohistochemical study. Am J Clin Pathol 125:399-406.

31. Catalano MG, Fortunati N, Pugliese M, Marano F, Ortoleva L, Poli R, Asioli S, Bandino A, Palestini N, Grange C, Bus- solati B, Boccuzzi G 2012 Histone deacetylase inhibition modulates E-cadherin expression and suppresses migration and invasion of anaplastic thyroid cancer cells. J Clin Endocrinol Metab 97:E1150-E1159.

32. Kim T, Veronese A, Pichiorri F, Lee TJ, Jeon YJ, Volinia S, Pineau P, Marchio A, Palatini J, Suh SS, Alder H, Liu CG, Dejean A, Croce CM 2011 p53 regulates epithelial-mesenchymal transition through microRNAs targeting ZEB1 and ZEB2. J Exp Med 208:875-883.

33. Chang CJ, Chao CH, Xia W, Yang JY, Xiong Y, Li CW, Yu WH, Rehman SK, Hsu JL, Lee HH, Liu M, Chen CT, Yu D, Hung MC 2011 p53 regulates epithelial-mesenchymal transition and stem cell properties through modulating miRNAs. Nat Cell Biol 13:317-323.

34. Knauf JA, Sartor MA, Medvedovic M, Lundsmith E, Ryder M, Salzano M, Nikiforov YE, Giordano TJ, Ghossein RA, Fagin JA 2011 Progression of BRAF-induced thyroid cancer is associated with epithelial-mesenchymal transition requiring concomitant MAP kinase and TGFbeta signaling. Oncogene 30:3153-3162.

35. Leboeuf R, Baumgartner JE, Benezra M, Malaguarnera R, Solit D, Pratilas CA, Rosen N, Knauf JA, Fagin JA 2008 BRAFV600E mutation is associated with preferential sensitivity to mitogenactivated protein kinase kinase inhibition in thyroid cancer cell lines. J Clin Endocrinol Metab 93:2194-2201.

36. Petti C, Molla A, Vegetti C, Ferrone S, Anichini A, Sensi M 2006 Coexpression of NRASQ61R and BRAFV600E in human melanoma cells activates senescence and increases susceptibility to cell-mediated cytotoxicity. Cancer Res 66:6503-6511.

37. Salerno P, Garcia-Rostan G, Piccinin S, Bencivenga TC, Di Maro G, Doglioni C, Basolo F, Maestro R, Fusco A, Santoro M 2001 TWIST1 plays a pleiotropic role in determining the anaplastic thyroid cancer phenotype. J Clin Endocrinol Metab 96:E772-E781.

38. Ralhan R, Cao J, Lim T, Macmillan C, Freeman JL, Walfish PG 2010 EpCAM nuclear localization identifies aggressive thyroid cancer and is a marker for poor prognosis. BMC Cancer 10:331.

39. Maetzel D, Denzel S, Mack B, Canis M, Went P, Benk M, Kieu C, Papior P, Baeuerle PA, Munz M, Gires O 2009 Nuclear signalling by tumour-associated antigen EpCAM. Nat Cell Biol 11:162-171.

40. Biddle A, Liang X, Gammon L, Fazil B, Harper LJ, Emich H, Costea DE, Mackenzie IC 2011 Cancer stem cells in squamous cell carcinoma switch between two distinct phenotypes that are preferentially migratory or proliferative. Cancer Res 71:5317.

Address correspondence to: Yuji Nagayama, $M D$

Department of Molecular Medicine Atomic Bomb Disease Institute Nagasaki University Graduate School of Biomedical Sciences 1-12-4 Sakamoto Nagasaki 852-8523 Japan

E-mail: nagayama@nagasaki-u.ac.jp 